\title{
Conditional Constellation Based-Distinct Native Attribute (CB-DNA) Fingerprinting for Network Device Authentication
}

\author{
Timothy J. Carbino, Michael A. Temple, and Juan Lopez Jr. \\ US Air Force Institute of Technology \\ Wright-Patterson AFB, Ohio 45433 USA \\ Email: [timothy.carbino, michael.temple, juan.lopez.ctr] @afit.edu
}

\begin{abstract}
Hackers have multiple avenues for accessing Industrial Control System (ICS) and can adversely impact network hardware, operating systems, and executables. This includes attacking hardware/software switches commonly used in waste water, water treatment, and power substation facilities. Unauthorized network intrusion can be mitigated by augmenting Media Access Control (MAC) based device identity (ID) verification processes using Physical-Layer (PHY) features. PHY augmentation is addressed here using Constellation Based Distinct Native Attribute (CB-DNA) features derived from unintentional Ethernet cable emissions. Collected emission symbols are mapped to a gradient-based binary constellation space where, for the first time, conditional constellation symbol features are used for device ID verification. Serial number discrimination is assessed using 16 devices from 4 different manufactures, with 12 serving as authorized devices and 4 (one from each manufacturer) serving as attacking rogue devices. Collectively considering 12,288 rogue attack scenarios using 256 verification models, the proposed CBDNA method is promising and yielded average Rogue Reject Rates (RRR) of $\mathbf{8 5 . 2 \%}<\mathbf{R R R}<\mathbf{9 3 . 1 \%}$ at $S N R \geq \mathbf{2 6 . 0} \mathbf{d B}$ using two network access criteria.
\end{abstract}

\section{INTRODUCTION}

Historically, system security has not been a top priority of cyber system developers, with integration of security measures often being an afterthought. These measures are not wellincorporated, are often addressed in patchwork updates, and leave weak points in the system that hackers can exploit. Between 2009 and 2011, the United States (US) infrastructure saw a "17-fold increase in computer attacks" [1]. At about this same time, the Industrial Control Systems (ICS) community, including the US and many other countries, began modernizing their legacy systems to use Internet Protocol (IP)-based solutions to control critical communications [2], [3]. Unfortunately, the lack of security emphasis during development of many IPbased solutions resulted in security weaknesses migrating into the ICS arena [4].

Adversaries have multiple attack vectors into ICS platforms, including 1) direct access to equipment by non-essential personnel, 2) open wireless and wired access ports [5], [6], and 3) remote user/device security and verification were not considered during system design [3], [6]. Thus, hackers can exploit these vulnerabilities to gain access to network hardware, operating systems, and/or executables [6].
To minimize these attack vectors Physical-Layer (PHY)based security measures can be employed to augment existing network authentication and security measures. Minute variations in the PHY's modulated responses are extracted to create unique, device-dependent fingerprints for each authorized device. Once features are discovered, the resultant fingerprints can be used to 1) classify signals as originating from one of a given number of authorized devices, or 2) verify signals are originating from a specific authorized device matching the claimed identity and not from an unauthorized/rogue device.

Wired and wireless network access control share some common vulnerabilities, e.g., Internet Protocol (IP) and Media Access Control (MAC) spoofing, that enable unauthorized network entry and only one compromised network connection can put all other connections at risk [13]. The use of PHY attributes to augment network security has been widely investigated for a variety of wireless communication devices [7]-[15]. However, much less attention has been given to PHY augmentation techniques for wired communication devices [16]-[19]. Closely related work in [16], performs device fingerprinting using Ethernet preamble correlation via a direct connection to the network card.

A comprehensive review of wireless PHY-based fingerprinting in [8] concluded that 1) work remains to be done in feature selection and extraction to improve device classification and ID verification, and 2) many PHY-based works do not perform an adequate performance evaluation given they only consider classification error not rouge rejection - only four of the ten papers reviewed in [8] considered device ID verification. This is one of the motivating factors for this work.

The first limitation in [8] was addressed in [18], [19] by using signal response transformation, alternate feature types, and conditional fingerprint features. Specifically, the classification work in [18] introduced a Constellation-Based Distinct Native Attribute (CB-DNA) feature generation method that projects unintentional Ethernet cable emissions (communication symbols) into a binary constellation space that enables both bit estimation and CB-DNA feature extraction from binary symbol clusters. Work in [18] was expanded in [19] to include classification using CB-DNA features extracted from conditional constellation sub-clusters, with improvements of $15 \%$ to $20 \%$ in average percent correct classification realized. 
The second limitation in [8] is addressed in this work using Rogue Reject Rates (RRRs) given that authorized device classification performance alone does not provide the community with a reliable performance assessment; assessment of RRR is of interest for network authentication. For rogue assessments, an unknown rogue device presents false bit level credentials matching an authorized device and is either 1) errantly granted access at the Rogue Accept Rate (RAR=1-RRR), or 2) correctly denied access at the RRR — rate is measured relative to either a specific number of device access attempts or the total number of bursts presented by a device.

Results in Sect. IV expand upon work in [19] by performing rogue ID verification assessments. This is done using a total of 16 Ethernet cards, four each from four different manufactures, with rogue assessments including various permutations of $N_{A}=12$ authorized and $N_{R}=4$ (one per manufacturer) rogue devices. Results include assessment of 12,288 rogue attack scenarios using 256 verification models.

The paper is structured as follows: Sect. II provides background information on the experimental setup and CB-DNA device fingerprinting. Section III discusses device discrimination, model development, $K$-fold cross-validation, and device ID verification. Device ID verification results are presented in Sect. IV followed by a summary and conclusions in Sect. V.

\section{BACKGROUND}

To motivate the device ID verification work here, background information is first provided on prior CB-DNA device discrimination works [18], [19] given that results herein are a direct extension. The section describes the signal collection process and construction of the device fingerprints.

\section{A. Collection Process}

A LeCroy oscilloscope with a near-field probe was used to collect 10BASE-T signals from a category 6 Ethernet cable connecting two computers. Relative probe-cable orientation was held constant during the collection process on all 16 network cards. MATLAB ${ }^{\circledR}$ was used to perform all postcollection processing. More information on the experimental collection process can be found in [20].

\section{B. $C B-D N A$ Fingerprinting}

Earlier related work in CB-DNA [18], [19] shows the development of a binary constellation from unintentional 10BASE-T Ethernet cable emissions and verified using bit error rate testing. The generation of a constellation for each device was accomplished through a symbol projection mechanism that transformed each communication symbol into a constellation point for a given burst (Ethernet frame). An example constellation is provided in Fig. 1, where the dashed line is the symbol estimation boundary. Projections to the left are estimated to be a zero, otherwise a one.

The statistical features extracted from constellation space DNA, stem from variations within the network cards PHY and subsequently supports device fingerprinting. The device verification process used here relies on the CB-DNA fingerprints

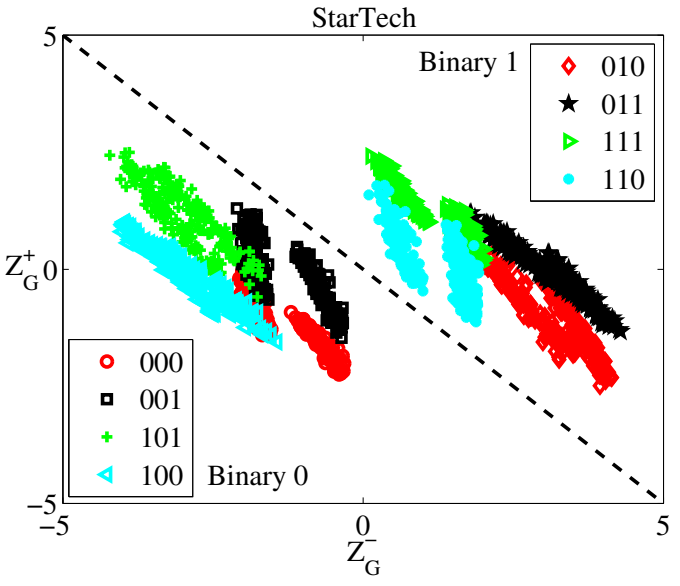

Fig. 1. Binary constellation diagram, symbol estimation boundary and conditional sub-cluster regions for card manufacturer StarTech (M3). The legend highlights the eight possible three-bit sequences where the center bit in each sequence represents the current estimated bit.

that contain conditional sub-clusters derived from within the original binary constellation. These conditional sub-clusters come from the eight possible bit combinations that can precede and succeed the bit being estimated i.e., bit combinations of $\left[\begin{array}{lll}0 & \mathbf{X} & 0\end{array}\right],\left[\begin{array}{lll}0 & \mathbf{X} & 1\end{array}\right],\left[\begin{array}{lll}1 & \mathbf{X} & 0\end{array}\right]$ and $\left[\begin{array}{lll}1 & \mathbf{X} & 1\end{array}\right]$, where $\mathbf{X}$ denotes the bit being estimated [19]. The sub-clusters within a constellation are highlighted in Fig. 1 based on the eight possible bit combinations for three consecutive bits.

Calculation of statistical CB-DNA fingerprint features originate within designated cluster/sub-cluster regions for a total of $N_{C R}=10$, using the mean $(\mu)$, variance $\left(\sigma^{2}\right)$, skewness $(\gamma)$, kurtosis $(\kappa)$, covariance $(C o v)$, coskewness $\left(\beta_{1 \times 2}\right)$, and cokurtosis $\left(\delta_{1 \times 3}\right)$. A Regional Cluster Fingerprint $F_{R_{i}}^{C B}$ given by (1), where the superscripted $-/+$ sign denotes constellation dimension $\left(Z_{G}^{-}\right.$and $Z_{G}^{+}$in Fig. 1) and $i=1,2, \ldots, N_{C R}$. The final Composite $C B$-DNA Fingerprint $F_{C}^{C B}$ dimension is $1 \times\left(14 \times N_{C R}\right)$ and is constructed by concatenating $F_{R_{i}}^{C B}$ from (1) as in (2) [18]. Verification assessment uses a total of $N_{\text {Feat }}=140$ CB-DNA fingerprint features.

$$
\begin{gathered}
F_{R_{i}}^{C B}=\left[\mu_{R_{i}}^{-}, \mu_{R_{i}}^{+}, \sigma_{R_{i}}^{2-}, \sigma_{R_{i}}^{2+}, \gamma_{R_{i}}^{-}, \gamma_{R_{i}}^{+}, \kappa_{R_{i}}^{-}, \kappa_{R_{i}}^{+},\right. \\
\left.\operatorname{Cov}_{R_{i}}, \beta_{1 \times 2}^{R_{i}}, \delta_{1 \times 3}^{R_{i}},\right]_{1 \times 14} \\
F_{C}^{C B}=\left[F_{R_{1}}^{C B}: F_{R_{2}}^{C B}: F_{R_{3}}^{C B}: \cdots: F_{R_{N_{C R}}^{C B}}^{C B}\right]_{1 \times\left(14 N_{C R}\right)}
\end{gathered}
$$

\section{ExPERIMENTAL Methodology}

This section addresses device discrimination, classification model development for verification to include $K$-fold crossvalidation, and verification process details.

An important aspect of device discrimination is assessment under varying channel conditions, i.e., at varying Signal-toNoise Ratio $(S N R)$. Experimentally collected signals averaged an $S N R$ of $S N R_{C} \approx 30 \mathrm{~dB}$. To assess channel variation effects, a total of $N_{N z}=6$ independent like-filtered Additive White Gaussian Noise (AWGN) realizations were added to 
the collected signals to achieve analysis $S N R_{A} \in\left[\begin{array}{ll}12 & 32\end{array}\right] \mathrm{dB}$ in $2 \mathrm{~dB}$ steps $\left(S N R\right.$ is used in place of $S N R_{A}$ henceforth for brevity). Each AWGN realization was generated, filtered, power-scaled and added to the collected signal responses. This process was repeated for $N_{S}=1000$ collected signal responses per card to generate a total of $N_{F}=N_{S} \times N_{N z}=6000$ fingerprints for model development and device verification.

\section{A. Model Development}

A total of $N_{C}=16$ classes are used with each class being represented by one of four devices from one of four different manufactures to include D-Link (M1), Intel (M2), TRENDnET (M3), and StarTech (M4). Table I lists the ManufacturerDevice (M:D) references. To assess RRR performance, a subset of devices is held-out from model development. Consequently, the set of 16 possible devices was divided into two disjoint sets representing $N_{A(i)}=12$ authorized devices and $N_{R(i)}=4$ rogue devices where $i=1,2,3, \ldots, 256$ denotes permutation number.

Each of the $N_{R(i)}$ rogue sets contain 1-of-4 devices from each manufacturer and are selected as four-choose-one, yielding a total of 256 possible rogue Perm sets. Accordingly, the $N_{A(i)}$ authorized sets contain the remaining 3-of-4 devices from each manufacturer. Table I provides ten representative permutations where, $\mathrm{X} \in N_{A(i)}$ and $\{\mathrm{R} 1, \mathrm{R} 2, \mathrm{R} 3, \mathrm{R} 4\} \in N_{R(i)}$. For each permutation, all $N_{R(i)}=4$ rogues present false credentials matching each of the $N_{A(i)}=12$ authorized devices, for a total of $4 \times 12=48$ rogue scenarios per permutation.

The Multiple Discriminant Analysis, Maximum Likelihood (MDA/ML) model development is adopted from prior related work [19], [21]. The $K$-fold cross-validation steps are [22]: 1) divide the training fingerprints $N_{T n g}$ into $K$ equal size disjoint blocks of $N_{T n g} / K, 2$ ) hold out one block and train on $K-1$ blocks to produce projection matrix $\mathbf{W}$, and 3) use

TABLE I

MANUfaCturer-Device (M:D) COMBinations USED For VERIFICATION ASSESSMENTS. SHOWING 10 OF 256 PERMUTATIONS WITH X DENOTING $N_{A(i)}=12(i=15,16,82,113,114,143,144,159,226$ AND 242) Authorized Devices And 4 Rogue Devices (R1, R2, R3, R4).

\begin{tabular}{|c|c|c|c|c|c|c|c|c|c|c|c|}
\hline \multirow{2}{*}{ Reference } & \multirow{2}{*}{$\begin{array}{c}\text { MAC Address } \\
\text { Last Four }\end{array}$} & \multicolumn{10}{|c|}{ Perm $(i)$} \\
\hline & & 15 & 16 & 82 & 113 & 114 & 143 & 144 & 159 & 226 & 242 \\
\hline M1:D1 & D966 & R1 & R1 & $x$ & $x$ & $x$ & $x$ & $x$ & $x$ & $X$ & $x$ \\
\hline M1:D2 & DA06 & $x$ & $x$ & R1 & $\mathrm{R} 1$ & R1 & $X$ & $\mathrm{X}$ & $X$ & $x$ & $x$ \\
\hline M1:D3 & DA07 & $X$ & $X$ & $X$ & $X$ & $X$ & $\mathrm{R} 1$ & R1 & R1 & $x$ & $x$ \\
\hline M1:D4 & $60 \mathrm{E} 0$ & $X$ & $x$ & $x$ & $x$ & $x$ & $x$ & $x$ & $x$ & $\mathrm{R} 1$ & $\mathrm{R} 1$ \\
\hline M2:D1 & 1586 & $\mathrm{R} 2$ & R2 & $X$ & $x$ & $X$ & R2 & R2 & $X$ & $x$ & $X$ \\
\hline M2:D2 & $1 \mathrm{~A} 93$ & $x$ & $x$ & R2 & R2 & $X$ & $X$ & $x$ & R2 & $X$ & $x$ \\
\hline M2:D3 & 1A59 & $x$ & $x$ & $x$ & $X$ & $X$ & $X$ & $X$ & $x$ & $\mathrm{R} 2$ & $x$ \\
\hline M2:D4 & $1 \mathrm{~A} 9 \mathrm{E}$ & $X$ & $x$ & $x$ & $x$ & $\mathrm{R} 2$ & $x$ & $x$ & $x$ & $x$ & $\mathrm{R} 2$ \\
\hline M3:D1 & $9 \mathrm{~B} 55$ & $X$ & $X$ & R3 & R3 & R3 & X & $X$ & $X$ & R3 & R3 \\
\hline M & & $X$ & $X$ & $X$ & $X$ & $X$ & $X$ & $X$ & X & $X$ & $X$ \\
\hline M3:D3 & $9 B 54$ & $x$ & X & $\mathrm{X}$ & X & $x$ & $x$ & $x$ & $X$ & 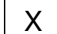 & X \\
\hline M3:D4 & 9B56 & R3 & R3 & $x$ & $X$ & $X$ & R3 & R3 & R3 & $X$ & $x$ \\
\hline M4:D1 & $32 \mathrm{CB}$ & $x$ & $x$ & $x$ & $\mathrm{R} 4$ & $x$ & $x$ & $x$ & $x$ & $x$ & $x$ \\
\hline M4:D2 & $32 \mathrm{~B} 4$ & $x$ & $X$ & $\mathrm{R} 4$ & $x$ & R4 & X & $X$ & $X$ & $\mathrm{R} 4$ & $\mathrm{R} 4$ \\
\hline & $96 \mathrm{~F} 4$ & R4 & $x$ & $X$ & $X$ & $x$ & $\mathrm{R} 4$ & $x$ & R4 & $x$ & $X$ \\
\hline M4:D4 & 3048 & $x$ & R4 & $x$ & $x$ & $x$ & $x$ & R4 & $x$ & $x$ & $x$ \\
\hline
\end{tabular}

the holdout block and $\mathbf{W}$ for validation. The $\mathbf{W}$ from the best training iteration is output and used as the MDA/ML model for device ID verification assessment. The process was repeated to generate an $S N R$-dependent $\mathbf{W}(S N R)$ for each $S N R$.

Summarizing, each MDA/ML model developed for device ID verification is based on the following: 1) $N_{A(i)}=12$ authorized classes, 2) $K=5$ cross-fold validation, 3) $N_{F}=6,000$ total fingerprints, 4) an equal number of Training $\left(N_{T n g}=N_{F} / 2\right)$ and Testing $\left(N_{T s t}=N_{F} / 2\right)$ fingerprints for authorized devices, and 5) $N_{T s t}=6000$ fingerprints for each rogue device.

\section{B. Device ID Verification}

Device ID verification is a 1 versus 1 comparison for assessing how much a fingerprint for a claimed identity (reference) looks like a fingerprint for the actual identity. The ID verification steps include: 1) developing a reference model, 2) selecting a similarity measure, 3) determining devicedependent threshold values $t_{V}(d)(d=1,2, \ldots, 12)$ based on Probability Mass Function (PMF) characteristics and desired True Verification Rate (TVR) and False Verification Rate (FVR) performance, 4) generating a test statistic $Z_{V}$ for each unknown fingerprint from the device presenting the claimed ID, 5) comparing test statistic $Z_{V}$ with threshold $t_{V}(d)$ according to (3) and making a final decision to Grant network access (accept) or Deny network access (reject).

$$
\begin{aligned}
& Z_{V} \leq t_{V}(d) \Rightarrow \text { Grant } \\
& Z_{V}>t_{V}(d) \Rightarrow \text { Deny }
\end{aligned}
$$

MDA/ML model generation occurred for all 256 permutations of $N_{A(i)}=12$ (of 16) and associated disjoint subset $N_{R(i)}=4$. Accounting for $N_{A(i)}=12$ authorized devices and 256 rogue permutations of $N_{R(i)}=4$ rogue devices provides a total of $12 \times 256 \times 4=12,288$ possible rogue assessment scenarios. Due to space limitations, it is not possible to present all rogue assessment scenarios. A reduced set of permutations were examined based on the percent correct classification $\% C$ for all 256 permutations over the range of SNR. Fig. 2 demonstrates a periodic trend, attributed to how devices were assigned to each permutation, in $\% C$ for each $S N R$ over all permutations with three specific $S N R$ 's highlighted.

The evaluation of the $\% C$ over all permutations yielded no statistical outliers, Therefore, the representative $N_{A(i)}$ permutations in Table I were chosen for presentation given they are statistically representative of highest $(i=15,16,143,144$, and $159)$ and lowest $(i=82,113,114,226$, and 242) classification performance $\% C$ at $S N R=18 \mathrm{~dB}$ and are subsequently used for rogue assessment in Sect. IV.

Euclidean distance was chosen as the similarity measure for device verification. Then test statics are calculated for the $N_{T n g}=3000$ and $N_{T s t}=3000$ fingerprints to generate the authorized device PMFs for the training and testing sets. The generated PMFs are then used to create the Receiver Operating Characteristic (ROC) curves in Sect. IV which provide a measure of system performance. The ROC curves are used to set device dependent threshold values, $t_{V}(d)$ for 


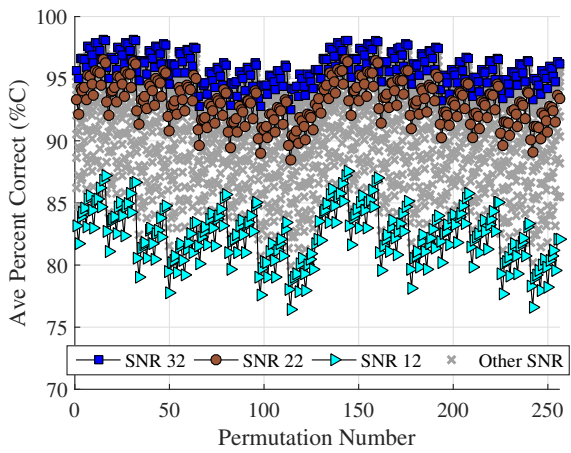

Fig. 2. CB-DNA Average Cross-Class $\% C$ for $N_{A(i)}=12$ Class MDA/ML Discrimination Using All 256 Permutations $(i=1,2, \ldots, 256)$ of 16 Devices Taken 12 at a Time.

$d=1,2, \ldots, N_{A(12)}$, which are arbitrarily set here for TVR=0.9. Individual test statistics $Z_{V}$ also used to generate Burst-byBurst (BbB) network access attempts in Sect. IV.

The rogue assessment is completed by inputting an unknown fingerprint and generating a test statistic $Z_{V}$ and comparing it against the threshold for the device it claims to be. The device is either accepted or rejected based on $Z_{V}$ and $t_{V}(d)$ according to (3). This process is repeated for all $N_{\text {Tst }}=6000$ rogue fingerprints against each of the $N_{A}=12$ authorized devices providing a total of $6,000 \times 12=72,000$ tests performed per rogue device for a given rogue assessment. Individual test statistics and ROC curves are also used in Sect. IV to highlight system performance.

\section{VERIFICATION RESUlTS}

Device ID verification is assessed here using two network access methods, including 1) ROC curves for making Binary Grant/Deny (BGD) decisions using test statistic PMFs, and 2) stem plots of raw Euclidean distance $Z_{V}$ for Burst-by-Burst (BbB) decisions.

Assessment of BGD verification performance is accomplished using ROC curves which are generated here as TVR vs. FVR and TVR vs. RAR for authorized and rogue devices, respectively. The TVR vs. RAR presentation is a matter of convenience and enables 1) direct assessment of rogue performance for a given authorized device TVR (vertical displacement in Fig. 3(a) and Fig. 3(b) are identical), and 2) easy calculation of $R R R=1-R A R$. The PMFs used to generate Fig. 3 ROC curves are based on independent test statistics $Z_{V}$ generated per Sect. III-B and include a total of $N_{T s t}=3000$ and $N_{T s t}=6000$ fingerprints per authorized and rogue device, respectively.

For ROC curves in Fig. 3, BGD success is derived from arbitrarily defined criteria that includes: 1) Authorized Device verification of TVR $>0.9$ and FVR $<0.1$ in Fig. 3(a), with the Authorized Accept Rate (AAR), provided in Tables II and III, being the number of authorized access attempts satisfying this criteria divided by the total number of attempts, and 2) Rogue Device verification of $\mathrm{TVR}>0.9$ and $\mathrm{RAR}<0.1$ in Fig. 3(b), with RRR being the number of rogue access attempts satisfying this criteria divided by the total number of attempts.
The common TVR $>0.9$ benchmark is shown as a horizontal dotted line in Fig. 3, with curves for successful BGD attempts denoted by solid lines and failures denoted by dashed curves.

The solid ROC curves in Fig. 3(a) show that all $N_{A}=12$ authorized devices meet or exceed the arbitrary TVR $>0.9$ and FVR $<0.1$ criteria at $S N R=18 \mathrm{~dB}$ for Perm 15 and are granted network access (AAR $=100 \%)$. Similarly, ROC curves in Fig. 3(b) at the same TVR $>0.9$ show rogue R3 successfully rejected in 7 of 12 attempts (solid curves, RRR=58\%) and gaining access in 5 of 12 attempts (dashed curves).

The $\mathrm{BbB}$ verification process for authorized devices is illustrated in Fig. 4(a) which shows $N_{T s t}=3000$ Euclidean distance $Z_{V}$, where blue O's denote access being granted correctly and red X's denote access being denied incorrectly, from all authorized devices (A1-A12). The device dependent verification thresholds $t_{V}(d)$ are indicated by a solid black horizontal line and correspond to TVR $=90 \%$ operating points in Fig. 3(a) ROC curves.

The $\mathrm{BbB}$ verification process for rogue devices is illustrated in Fig. 4(b) which shows $N_{T s t}=6000$ Euclidean distance $Z_{V}$, where blue O's denote access being correctly denied and red $X$ 's denote access being incorrectly granted, per scenario, with R3 falsely claiming each of the authorized device IDs (R3:A1, R3:A2, .., R3:A12). The authorized device $t_{V}(d)$ correspond to those in Fig. 4(a) and are used to make BbB decisions. In this case, RRR for $d^{\text {th }}$ claimed ID is calculated as the number of $Z(d)_{V}>t_{V}(d)$ divided by the total number of $Z(d)_{V}$ as provided in Tables II and III. It is clear in Fig. 4(b) that R3 (an M3 device) is least similar to A4, A5, and A6 (M2 devices) and A10, A11, and A12 (M4 devices) given the corresponding $Z_{V}$ are well above $t_{V}(d)$ for those devices. Of note are the authorized devices (A1, A3, A7, A8 and A9) that R3 appears most similar to in Fig. 4(b) which correspond to the 5 unacceptable scenarios (dashed ROC curves) in Fig. 3(b).

TABLE II

PERM 15 DEVICE ID VERIFICATION PERFORMANCE FOR Binary Grant/Deny (BGD) NETWORK ACCESS THAT INCLUDES (A) AN AUTHORIZED ACCESS RATE (AAR) OF AAR $=100 \%$ FOR $S N R \geq 18 \mathrm{~dB}$, AND (B) A BEST CASE ROGUE REJECTION RATE OF RRR $=87.5 \%$ AT $S N R \geq 26 \mathrm{~dB}$. RESULTS FOR Burst-by-Burst (BbB) ASSESSMENTS SHOW $\mathrm{RRR} \geq 87.8 \%$ AT $S N R \geq 18 \mathrm{~dB}$.

\begin{tabular}{|c|c|c|c|}
\hline \multirow{2}{*}{ SNR (dB) } & \multicolumn{2}{|c|}{ BGD } & BbB \\
\cline { 2 - 4 } & AAR (\%) & RRR (\%) & RRR (\%) \\
\hline 12 & 33.3 & 68.6 & 81.2 \\
\hline 14 & 41.7 & 72.9 & 83.6 \\
\hline 16 & 75.0 & 77.1 & 86.1 \\
\hline 18 & $\mathbf{1 0 0}$ & 77.1 & 87.8 \\
\hline 20 & $\mathbf{1 0 0}$ & 79.2 & 89.2 \\
\hline 22 & $\mathbf{1 0 0}$ & 81.3 & 90.3 \\
\hline 24 & $\mathbf{1 0 0}$ & 83.3 & 90.9 \\
\hline 26 & $\mathbf{1 0 0}$ & $\mathbf{8 7 . 5}$ & 91.5 \\
\hline 28 & $\mathbf{1 0 0}$ & $\mathbf{8 7 . 5}$ & 91.8 \\
\hline 30 & $\mathbf{1 0 0}$ & $\mathbf{8 7 . 5}$ & 92.1 \\
\hline 32 & $\mathbf{1 0 0}$ & $\mathbf{8 7 . 5}$ & 92.4 \\
\hline
\end{tabular}




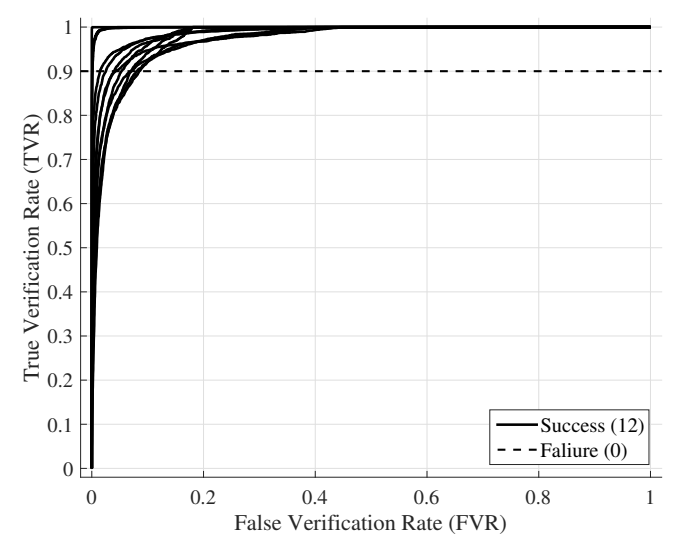

(a) Authorized Device ID Verification

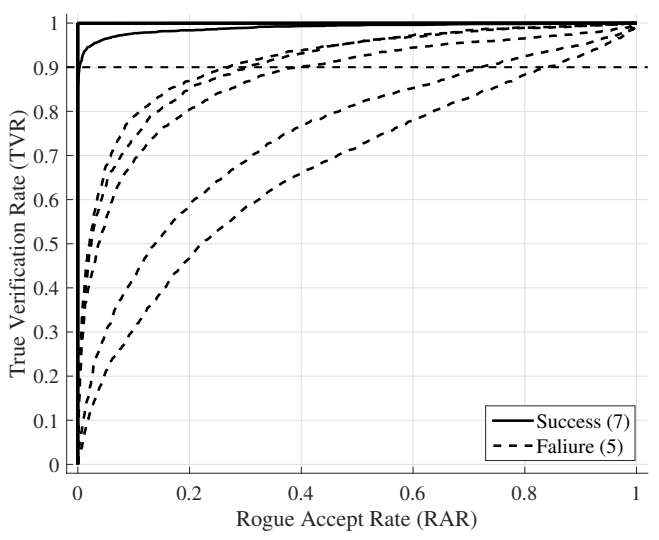

(b) Rogue Device (R3) ID Verification

Fig. 3. ID Verification ROC curves for Perm 15 in Table I at $S N R=18 \mathrm{~dB}$ utilizing a Euclidean distance measure of similarity. Relative to BGD network access decisions: (a) Authorized device success is AAR=100\% (12 of 12) for TVR $>0.9$ and FVR $<0.1$ criteria, and (b) Rogue device R3 success is RRR=58\% (7 of 12) for $\mathrm{RAR}<0.1$ criteria; dashed lines reflect the 5 scenarios not meeting success criteria.

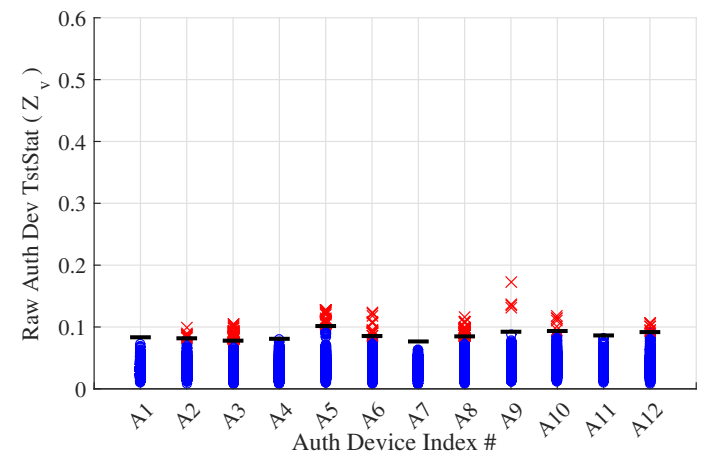

(a) Authorized Device (A1-A12) ID verification test statistics for $N_{\text {Tst }}=3000$ testing fingerprints per authorized device. Blue O's denote correctly granted access and red X's denote incorrectly denied access.

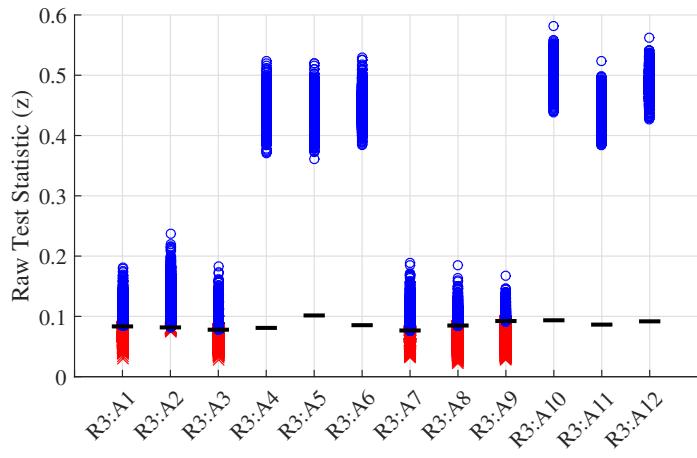

Rouge:Model Device Index \#

(b) Rogue Device (R3) verification test statistics for $N_{T s t}=6000$ testing fingerprints with R3 presenting a false ID for each authorized device (R3:A1-R3:A12). Blue O's denote correctly denied access and red X's denote incorrectly granted access

Fig. 4. Overlay of Euclidean distance test statistics $Z_{V}$ for Perm 15 devices at $S N R=18$ dB. Solid horizontal lines are device dependent $t_{V}(d)$ thresholds corresponding to ROC TVR=90\% in Fig. 3. Rogue results provide an indication of how much R3 "looks like" the authorized devices.

Results in Table II and Table III are presented as (\#Successes/Total\#Trials) $\times 100$ with bold entries denoting best or equivalent performance. For BGD results, AAR is based on $N_{A}=12$ authorized devices trials and RRR is based on $\left(N_{A}=12\right) \times\left(N_{R}=4\right)=48$ total trials. The BbB results are based on $\left(N_{A}=12\right) \times\left(N_{R}=4\right) \times\left(N_{T s t}=6000\right)=288,000$ trials.

Table II shows results for Perm 15 at all $S N R$ and highlights the direct relationship between $S N R$ and accept/reject rates. In addition, Table II shows that $S N R=18 \mathrm{~dB}$ is the lowest $S N R$ at which AAR $=100 \%$ for authorized devices; however, network access is granted in 11 of 48 rogue scenarios. Further investigation revealed that Rogue R3 (device M3:D4) was errantly granted network access in 5 of the 11 scenarios shown in Fig. 3(b). It is also evident in Table II that the BbB method consistency outperformed the BGD method.

Table III provides results for the 10 permutations listed in Table I and the average over all 12,288 rogue scenarios at $S N R=26 \mathrm{~dB}$. This $S N R$ is highlighted given the BGD RRR in Table II did not improve at higher $S N R$. BbB results collectively include $90 \%<\mathrm{RRR}<96 \%$ and of interest is that for the 10 permutations in Table III, the permutations yielding highest RRR had correspondingly poorer \%C than the permutations yielding lowest RRR-reflecting no direct relationship between classification and verification performance.

\section{SUMmary AND CONCLUSIONS}

This work addresses Physical-Layer (PHY) augmentation to MAC-based network authentication using device fingerprints composed of Constellation-Based Distinct Native Attribute (CB-DNA) features. Device ID verification was evaluated using a CB-DNA fingerprinting approach that maps unintentional wired emissions (communication symbols) into a 2D constellation space and then extracts higher-order statistics (features) from 10 cluster regions with conditional sub-clusters as a subset. Results are included for 256 rogue permutations of 16 available Ethernet cards, 4 each from 4 different man- 
TABLE III

ID Verification Results For 10 Selected Permutations at

$S N R=26 \mathrm{~dB}$ SHOWING AAR (3000 ATTEMPTS) AND RRR (6000

ATTEMPTS) FOR Binary Grant/Deny (BGD) NETWORK ACCESS. Burst-by-Burst (BbB) RRR (288,000 ATTEMPTS) IS ALSO SHOWN ALONG with Averages over All Permutations and all Rogue sCenarios.

\begin{tabular}{|c|c|c|c|c|}
\hline & \multirow[b]{2}{*}{ Perm \# } & \multicolumn{2}{|c|}{ BGD } & \multirow{2}{*}{$\begin{array}{c}\mathrm{BbB} \\
\mathrm{RRR}(\%)\end{array}$} \\
\hline & & AAR $(\%)$ & RRR $(\%)$ & \\
\hline \multirow{5}{*}{$\begin{array}{l}0 \\
0 \\
0 \\
\overrightarrow{0} \\
0 \\
0 \\
0\end{array}$} & 82 & 100 & 81.2 & 93.4 \\
\hline & 113 & 100 & 85.4 & 93.7 \\
\hline & 114 & 100 & 85.4 & 95.2 \\
\hline & 226 & 100 & 83.3 & 95.1 \\
\hline & 242 & 100 & 87.5 & 96.3 \\
\hline \multirow{5}{*}{ 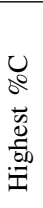 } & 15 & 100 & 87.5 & 91.5 \\
\hline & 16 & 100 & 85.5 & 91.8 \\
\hline & 143 & 100 & 85.4 & 91.3 \\
\hline & 144 & 100 & 85.4 & 90.9 \\
\hline & 159 & 100 & 83.3 & 90.7 \\
\hline \multicolumn{2}{|c|}{ ALL Perms } & 100 & 85.2 & 93.1 \\
\hline
\end{tabular}

ufacturers, with 12 cards serving as authorized devices and 4 cards serving as rogue devices. Euclidean distance was used as the measure of similarity for ID verification.

The CB-DNA device ID verification approach is promising and warrants further study. For $S N R \geq 26 \mathrm{~dB}$, overall performance includes: 1) average correct classification of $\% \mathrm{C} \geq 90 \%$ and Authorized Accept Rate (AAR) of AAR $=100 \%$ for authorized devices, and 2) an average Rogue Rejection Rate (RRR) of $85.2 \%<\mathrm{RRR}<93.1 \%$ across 12,288 rogue scenarios using Binary Grant/Deny and Burst-by-Burst accept/reject criteria. The majority of the rogue acceptances occurred between a rogue and its own manufacturing class. However, $100 \%$ of the acceptances occurring between a rogue and a different manufacturer happened between (D-Link and TRENDnET) which use the same LAN transformer [19]. This suggests that LAN transformer RF characteristics influences fingerprint features and impacts the ability to perform Ethernet card ID authentication across manufacturers. Ultimately, ID verification results for scenarios produced similar AAR and slightly better RRR relative to results in [16]. The main difference in the approaches is that here discrimination is based on signal constellation features versus correlation features. Benefits of the method here include: 1) only requiring external cable access and not individual twisted wire pairs inside the cable, 2) using sample rates that can be 4 to 10 times lower, and 3) operating at lower $S N R$ while still achieving desirable AAR and RRR.

Reliable device authentication is very important from an ICS network security perspective. The PHY approach presented provides away to authenticate any device that connects to an open wired access port at a ICS facility. Similarly, any device that is intrinsically part of the ICS system could be fingerprinted and used to later verify that no new devices have been introduced to the system. The CB-DNA verification results are encouraging and work continues to improve performance at various stages of the process by averaging multiple fingerprints prior to test statistic generation, and/or averaging multiple test statics prior to making grant/deny decisions. Furthermore, accumulation of symbols from multiple bursts prior to fingerprint generation has potential to increase performance.

\section{REFERENCES}

[1] D. Sanger and E. Schmitt, "Rise is seen in cyberattacks targeting us infrastructure," The New York Times, vol. 26, 2012.

[2] N. Cai, J. Wang, and X. Yu, "Scada system security: Complexity, history and new developments," in Industrial Informatics, 2008. INDIN 2008. 6th IEEE International Conference on, July 2008, pp. 569-574.

[3] K. Stouffer, J. Falco, and K. Scarfone, "Guide to industrial control systems (ics) security," NIST special publication, pp. 800-82, 2011.

[4] S. Gold, "The scada challenge: securing critical infrastructure," Network Security, vol. 2009, no. 8, pp. 18-20, 2009.

[5] J. Stamp, J. Dillinger, W. Young, and J. DePoy, "Common vulnerabilities in critical infrastructure control systems," SAND2003-1772C. Sandia National Laboratories, 2003.

[6] H. Okhravi and D. Nicol, "Applying trusted network technology to process control systems," in Critical Infrastructure Protection II. Springer, 2008, pp. 57-70.

[7] B. Danev, H. Luecken, S. Capkun, and K. El Defrawy, "Attacks on Physical-Layer Identification," in Proc of the third ACM Conf on Wireless network security. ACM, 2010, pp. 89-98.

[8] B. Danev, D. Zanetti, and S. Capkun, "On Physical-Layer Identification of Wireless Devices," ACM Computing Surveys (CSUR), vol. 45, no. 1, p. 6, 2012.

[9] N. Hu and Y.-D. Yao, "Identification of legacy radios in a cognitive radio network using a radio frequency fingerprinting based method," in IEEE Int'l Conf on Communications (ICC), June 2012, pp. 1597-1602.

[10] Y. Huang and H. Zheng, "Radio Frequency Fingerprinting Based on the Constellation Errors," in Communications (APCC), 2012 18th AsiaPacific Conf on. IEEE, 2012, pp. 900-905.

[11] M. Edman and B. Yener, "Active Attacks Against Modulation-Based Radiometric Identification," Rensselaer Institute of Technology, Technical report, pp. 09-02, 2009.

[12] V. Brik, S. Banerjee, M. Gruteser, and S. Oh, "Wireless Device Identification with Radiometric Signatures," in Proc of the 14th ACM Int'l Conf on Mobile computing and networking. ACM, 2008, pp. 116-127.

[13] A. Candore, O. Kocabas, and F. Koushanfar, "Robust Stable Radiometric Fingerprinting for Wireless Devices," in Hardware-Oriented Security and Trust, 2009. HOST '09. IEEE Int'l Workshop on, July, pp. 43-49.

[14] M. Pospisil, R. Marsalek, and J. Pomenkova, "Wireless Device Authentication Through Transmitter Imperfections - Measurement and Classification," in Personal Indoor and Mobile Radio Communications (PIMRC), 2013 IEEE 24th Int'l Symp on, Sept 2013, pp. 497-501.

[15] S. Rehman, K. Sowerby, and C. Coghill, "Analysis of receiver front end on the performance of rf fingerprinting," in Personal Indoor and Mobile Radio Communications (PIMRC), 2012 IEEE 23rd Int'l Symp on, Sept 2012, pp. 2494-2499.

[16] R. M. Gerdes, M. Mina, S. F. Russell, and T. E. Daniels, "PhysicalLayer Identification of Wired Ethernet Devices," Information Forensics and Security, IEEE Trans on, vol. 7, no. 4, pp. 1339-1353, 2012.

[17] R. M. Gerdes, T. E. Daniels, M. Mina, and S. F. Russell, "Device Identification via Analog Signal Fingerprinting: A Matched Filter Approach," in NDSS, 2006.

[18] T. J. Carbino, M. A. Temple, and T. J. Bihl, "Ethernet Card Discrimination Using Unintentional Cable Emissions and ConstellationBased Fingerprints," in Computing, Networking and Communications (IWCNC), 2015 Int'l Workshop on, 2015.

[19] T. J. Carbino, M. A. Temple, and J. Lopez Jr, "A Comparison of PHY-Based Fingerprinting Methods Used to Enhance Network Access Control," in ICT Systems Security and Privacy Protection. Springer, 2015, pp. 204-217.

[20] T. J. Carbino and R. O. Baldwin, "Side Channel Analysis of Ethernet Network Cable Emissions," in 9th Int'l Conf on Cyber Warfare and Security, ICCWS-2014.

[21] B. W. Ramsey, M. A. Temple, and B. E. Mullins, "PHY Foundation for Multi-Factor ZigBee Node Authentication," in Global Communications Conf (GLOBECOM), 2012 IEEE, Dec 2012, pp. 795-800.

[22] R. O. Duda, P. E. Hart, and D. G. Stork, Pattern Classification. John Wiley \& Sons, 2012. 